\title{
Empirical evaluation of four-variate thematic maps for expert users
}

\author{
Korycka-Skorupa J. ${ }^{\text {a, }}$, Gołębiowska I. ${ }^{\text {b }}$ \\ ${ }^{a}$ Department of Geoinformatics, Cartography and Remote Sensing, Faculty of Geography and Regional Studies, University of \\ Warsaw,Poland, j.skorupa@uw.edu.pl \\ ${ }^{b}$ Department of Geoinformatics, Cartography and Remote Sensing, Faculty of Geography and Regional Studies, University of \\ Warsaw, Poland, i.golebiowska@uw.edu.pl \\ * Corresponding author
}

Keywords: Multivariate map; Thematic mapping; User study; Eye-tracking

\begin{abstract}
:
Multivariate mapping is a cartographic technique in which multiple data variables are encoded into a single map. A variety of design solutions for multivariate mapping refers to the number of phenomena mapped, the map type, and the graphic variables applied. The main purpose of multivariate maps is to compare spatial distribution and to indicate relation between visualized phenomena (Kraak et al. 2020).
\end{abstract}

As an advantage of multivariate maps it can be indicated that the amount of information conveyed by a single map can be increased compared to an univariate map (Nelson 2020). Moreover, relationships between two or more mapped phenomena can be found and recognized by a map user. Multivariate maps are also a compact form: instead of preparing a series of maps of the same area, one can choose to elaborate a single multivariate map. However, multivariate maps are challenging for users due to information complexity. Furthermore, they are seldomly applied in popular media; therefore, a potential user may not be experienced in using it. As Nelson (2020) noted, a map maker must thus balance the benefits of multivariate maps with the audience's ability and willingness to engage with the challenging map.

The multivariate mapping covers a wide variety of design solutions. For instance, Nelson (2000) and Elmer (2013) listed how visual variables are applied in different multivariate maps. Visual variables configuration has been empirically verified with regards to supporting different types of operations (e.g. filtering a separate phenomenon or searching for correlation between phenomena) (Nelson 2000). Multivariate maps were divided into intrinsic and extrinsic maps (Šašinka et al. 2021). The former refers to maps with variables shown separately on a single map, e.g. choropleth map with proportional symbols map. The latter - to maps with represented phenomena that are visually inseparable (e.g. bivariate choropleth map), and they were found to be less useful while empirically assessed by Šašinka et al. (2019, 2021).

The aim of this study is to evaluate three solutions of multivariate maps. Unlike other authors (mainly evaluating bivariate maps), we decided to compare three solutions when mapping four variables (Figure 1): two types of multivariate maps, intrinsic (Fig.1A) and extrinsic (Fig. 1B) and a simple univariate alternative (Fig. 1C). two types of multivariate maps (intrinsic and extrinsic) and a simple univariate alternative. We analysed usability performance metrics (answer time, answer accuracy and subjective rating of task difficulty) and eye-tracking data. We focused on the impact of task type on users' performance when comparing the map options. We hypothesised that one four-variate map would be better for complex tasks, whereas a univariate map would be suitable for simple tasks. Moreover, maps with separable variables applied should be more useful when data sets need to be accessed individually rather than when the task goal is to find data correlation.

We applied mixed factorial design with map type and task complexity as independent variables, and performance metrics (answer time, answer accuracy, eye-tracking metrics) as the dependent variable. We applied within-subject design. In total, 16 participants took part in the study. All of them have graduated with an MSc degree majoring in cartography. 93.3\% use and make maps in their professional work and use maps on a daily basis.

The collected results showed that experts used the maps with similar results for answer time and accuracy, even when using four-variable intrinsic maps, which is considered to be a challenging solution. However, eye-tracking data provided more nuances in relation to the difference in cognitive effort evoked by the tested maps across task types, especially with regards to fixation duration of map legends.

We believe the conducted study provides input into understanding how to design helpful multivariate maps that might be a useful tool when making decisions on different levels of complexity: from simple (requiring referring to a single variable) to complex ones (referring to many variables at once). We thus believe that further investigations and more empirical research, including specific groups of users and specific kinds of data, will enable us to further develop the understanding of multivariate mapping and usage. 


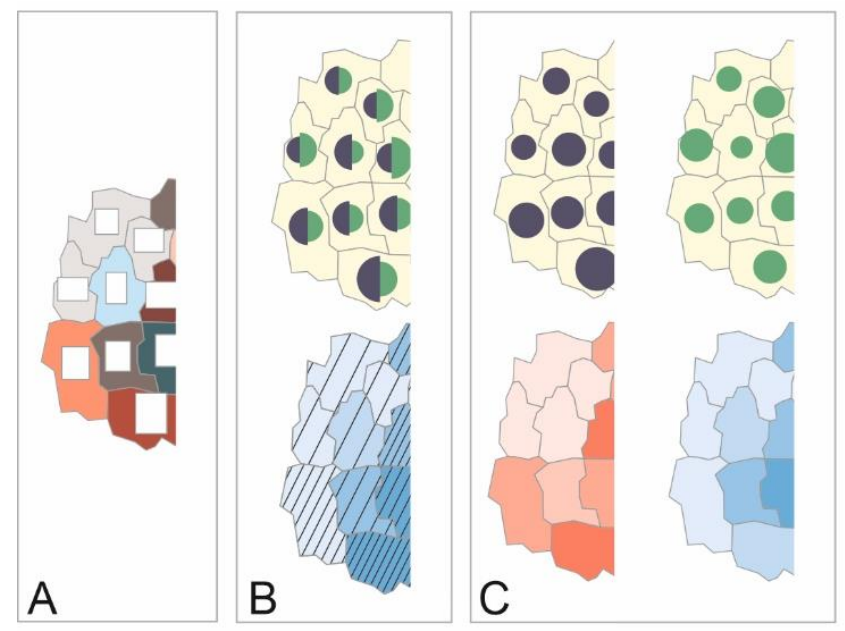

Figure 1. Examples of maps tested in the study. Source: own elaboration.

\section{References:}

Elmer, ME 2013, 'Symbol considerations for bivariate thematic maps', in Proceedings of the 26th International Cartographic Conference (ICC). International Cartographic Association: Dresden. Available from: http://icaci.org/files/documents/ICC_proceedings/ICC2013/_extendedAbstract/278_proceeding.pdf [08 June 2021]

Kraak, MJ, Roth, RE, Ricker, B, Kagawa, A, Le Sourd, G 2020, Mapping for a Sustainable World. The United Nations: New York.

Nelson, ES 2000 'The impact of bivariate symbol design on task performance in a map setting', Cartographica, vol. 37 , no. 4, pp. 61-78.

Nelson, J 2020, 'Multivariate mapping', The Geographic Information Science \& Technology Body of Knowledge, John P. Wilson (ed.). Available from: https://gistbok.ucgis.org/bok-topics/multivariate-mapping [15 June 2021].

Šašinka, Č, Stachoň, Z, Kubíček, P, Tamm, S, Matas, A \& Kukaňová, M 2019, 'The impact of global/local bias on tasksolving in map-related tasks employing extrinsic and intrinsic visualization of risk uncertainty maps', The Cartographic Journal, vol. 56, no. 2, pp. 175-191.

Šašinka, Č, Stachoň, Z, Čeněk, J, Šašinková, A, Popelka, S, Ugwitz P \& Lacko, D 2021, 'A comparison of the performance on extrinsic and intrinsic cartographic visualizations through correctness, response time and cognitive processing', PLoS ONE, vol. 16, no. 4.

\section{Acknowledgements:}

This work was supported by the National Science Centre, Poland under the grant no. UMO-2016/23/B/HS6/03846. 